\title{
Integration of genomic technologies for accelerated cancer drug development
}

\author{
Mark Basikํㅗㄴ Spyro Mousses ${ }^{1}$, and Jeffrey Trent ${ }^{2}$
}

BioTechniques 35:580-593 (September 2003)

\begin{abstract}
New technologies have greatly increased the scientist's ability to investigate complex molecular interactions that occur in cancer development and to identify genetic alterations and drug targets. However, these new capabilities have not accelerated drug development efforts; rather, they may be contributing to increased research and development costs because the large number of new drug targets discovered through genomics need to be investigated in great detail to characterize their putative functional involvement in the disease process. One solution to this bottleneck in functional analysis is the use of high-throughput technologies to produce efficient processes that can rapidly handle the large flood of information at every stage of disease. This review examines the use of new and emerging DNA, tissue, and live-cell transfection microarray technologies that can be used to discover, validate, and translate information resulting from the completion of the Human Genome Project.
\end{abstract}

\section{INTRODUCTION}

Most cancers develop through a slow, multistep process that involves an accumulation of genetic and epigenetic alterations that drive the malignant phenotype through a highly complex network of molecular interactions. The analysis of late-stage cancer cells shows that they have accumulated a wide range of genomic alterations that permeate all levels of the flow of genetic information. The analysis of cancer therefore requires increasingly sophisticated tools that are capable of assessing this genome-scale complexity. Fortunately, massively parallel technologies to interrogate the human genome have greatly increased our ability to investigate physiological processes, pharmacological responses, and pathological states. These capabilities are now routinely applied to study various aspects of cancer, including the identification of new genetic alterations and drug targets that reveal new opportunities for therapeutic intervention. However, these exciting data have not yet accelerated traditional cancer drug development efforts. In fact, the large number of unknown targets discovered from genomics has perhaps contributed more to the increased cost of research and development than to an increase in the clinical success rate of drug development efforts. One problem is that most new targets are largely unexplored and need to be investigated in great detail to determine their involvement in the actual disease process. The effort required to determine if a target is functionally appropriate and clinically relevant is currently not routinely implemented in high throughput. Consequently, the rate of gene discovery greatly exceeds the rate at which new targets are functionally and clinically validated, leading to a major bottleneck in the process of drug discovery.
One solution to using the brute force approach of forcing genomics to fit conventional strategies is to develop new paradigms to conform drug discovery to genomic-scale analysis. For example, true genome-based drug discovery can be achieved by creatively integrating high-throughput technologies to produce an efficient process that can handle the flow of genome-scale information at every stage of the process. In this review we describe several new technologies that have been applied to interrogate cancer genomes to find a variety of tumor-specific perturbations, and efforts to develop other technologies for rapidly moving those findings forward to the next step in drug discovery. Finally, we will focus on how integrating these with translational technologies for clinical and functional validation can get us closer to the goal of accelerating the development of new anticancer drugs.

\section{HIGH-THROUGHPUT TARGET IDENTIFICATION USING DNA MICROARRAYS}

DNA microarray analysis has revolutionized biology by allowing researchers to simultaneously survey the expression and sequence of genes at a genomic scale $(1,2)$. These technologies, and the emerging informatic strategies for analyzing microarray data, have not only increased the throughput of genetic analysis, but also allowed us to put such events into global perspective, giving us unprecedented insight into the workings of a cell (1-4). The following is a brief description of ways in which DNA microarrays have been utilized to monitor cancer-associated alterations in gene expression, gene copy number, and sequence. The impact of DNA microarrays on the process of drug development is highlighted.

${ }^{2}$ The Translational Genomics Research Institute, Phoenix, AZ, USA 


\section{Tumor Gene Expression Profiling Using DNA Microarrays}

Since cancer cells are the end products of complex genetic alterations and contain aberrant expression of multiple genes, it became clear early on that DNA microarrays were the ideal tool to investigate the genetic basis of the tumorigenic phenotype in different tumors and tumor types (5). Since the expression patterns vary among different tumor types, one of the most powerful applications of DNA microarrays turned out to be the discovery of gene sets that can be used for the diagnosis of cancers and the classification of tumors for prognostic and predictive purposes. Among the first examples of the power of DNA microarrays in enabling the appropriate diagnosis of different cancers was the study of acute leukemia by Golub et al. (6), clearly clustering clinical leukemia samples into the known subsets of acute myelogenous leukemia and acute lymphoblastic leukemia on the basis of differences in gene expression profiles. They validated their approach by classifying unknown samples into the correct subgroup. Interestingly, some of the genes that were selected from the 6817 genes on the oligonucleotide array as the most discriminating markers of the leukemia subgroup had previously been associated with the biology of leukemias while others had not.

Similarly, Perou et al. (7) used cDNA microarrays to produce a tumor classification for breast cancer in which distinct breast tumor subgroups were identified on the basis of unbiased gene clusters that turned out to be biologically relevant, such as the estrogen-positive cluster and the her 2 cluster. In their study, genes associated with cellular proliferation were coordinately expressed, and their expression was correlated with standard pathological indices of mitotic activity. Given such associations, it is evident that microarray-based tumor classification may theoretically be applied to the discovery of targets correlated with specific biological tumor features. The classification of melanomas by Bittner et al. (8) is an example of such an approach. Cluster analysis of 31 melanoma patients using cDNA microarrays revealed two distinct subgroups. Some of the discriminating genes were related to invasive ability and motility, which allowed the authors to predict that cell spreading and migration could be discordant between melanoma cluster groups. This hypothesis was verified using in vitro cellular migration and motility assays and led to the further investigation of one of the genes, wnt $5 a$, as a critical factor in regulating tumor motility and thus a putative drug target (9).

More recently, Hedenfalk et al. (10) were able to classify breast tumors based on genetic context (presence of the BRCAI or $B R A C 2$ germline mutation), thus further refining the classification of breast tumors while confirming the importance of expression profiling in differentiating between tumors of different origins. Several other papers have reported tumor classifications based on expression profiling in soft tissue sarcomas (11), lung cancers (12), and an increasing number of other tumors (13-15). These classifications of tumors were intended more for diagnostic and prognostic purposes. In a more recent study, cDNA microarrays were used to organize leukemic tumors into different categories, depending on their response to chemotherapy (16). A "drug response score" was elaborated based on the differential expression of 28 genes in the different groups and used to correctly predict individual response to the tested chemotherapy in $85 \%$ of cases. Thus gene expression profiles can be used to identify or reinforce tumor classification systems, the biological basis of which may lead to the identification of new candidate drug targets. On the other hand, gene expression profiles may be used to identify patterns predictive of response to therapy and thus facilitate the selection of the appropriate target patient population for clinical testing.

\section{Pharmacogenomics}

DNA microarrays can be used to directly interrogate tumor cells for the genes associated with response and toxicity of specific pharmacological agents. A whole-genome approach could therefore be applied to the search for individual markers of sensitivity and resistance to therapies. Both constitutive and drugselected genetic and gene expression variations may be used to assess the predisposition to therapeutic success and toxicity for different anticancer drugs. Several approaches are currently being used: $(i)$ to identify markers of chemosensitivity (predictive of therapeutic efficacy); (ii) to identify the molecular consequences of therapy that can be targets themselves either alone or in potentially synergistic combination with the anticancer drug under study; and (iii) to identify markers specific to stable drugresistant clones, which can be used to understand the mechanism of acquired resistance and as secondary targets in combination with the original drug. An example of the first approach is the linking of gene expression profiles of the NCI-60 cancer cell lines with activity profiles against 1400 tested compounds in these same 60 cell lines (17). Such database integration can allow the definition of drug sensitivity profiles, thus clustering the cell lines according to chemosensitivity and not tissue of origin or biology. Moreover, the impact of individual gene expression variability on chemosensitivity was revealed by the correlation between the expression of dihydropyrimidine dehydrogenase, the rate-limiting enzyme of uracil and thymidine metabolism, and sensitivity to 5-fluorouracil. In a follow-up study, gene expression-based classifiers of drug sensitivity or resistance were generated for 232 compounds and evaluated on independent sets of data with encouraging results (18). A different approach was used by the Rosetta group (19), which obtained characteristic drug signature patterns of gene expression from microarrays performed on yeast strains. The presence or absence of these signature patterns in drug-treated cells carrying a mutation in a gene encoding a putative target established whether that target was required to generate the drug signature. Not only can the pathways altered by drugs be identified and their real target validated, but also unintended gene expression effects of the drug can be resolved to uncover secondary drug targets. The same group later assembled a compendium of expression profiles of yeast deletion mutants and drug-treated yeast strains and used this database to identify a novel target of the drug dyclonine, whose target was previously unknown, by matching the expression profile resulting from treatment with this drug with expression profiles in the compendium (20).

\section{Analysis of Mechanism}

In many cases, mechanisms of disease and molecular 
pathways can be modeled from gene expression data. There have been many papers that attempted to extract such biological information from associative gene expression data using a variety of techniques. One approach involves the application of sophisticated mathematical tools to build models of networks of interactions from cDNA microarray expression data (21). A second approach involves using microarrays to characterize deletion mutant and drug-treated yeast strains by the Rosetta group, as mentioned above (20). A reference database of gene expression profiles of 300 distinct mutations and chemical treatments was successfully used to decipher functions of uncharacterized mutated genes. In a more traditional cancer research application, Mousses et al. (21) demonstrated that gene expression profiles could be used to characterize completely unknown mechanisms such as hormone therapy response and resistance in prostate cancer in vivo. In that study, it was discovered that an androgen responsive cluster of genes was mediating the therapy response and failure in a xenograft model system. Additionally, there was another set of genes that were altered in the hormone refractory prostate tumors, which identified a rapamycinsensitive signaling pathway. This revealed a new therapeutic strategy for treating advanced prostate cancer (21). Similarly, whole-genome profiling of gene expression can be mined to uncover known pathways of drug mechanism of action, resistance, and metabolism. In the aforementioned analysis of the NCI-60 cell line panel (17), analysis of the integrated database on the basis of gene-drug correlations revealed clustering of the drugs according to mechanism of action in most cases, with some interesting surprises. For instance, the five most active topoisomerase 1 inhibitors, which do not require activation, clustered together while the two prodrugs clustered in a distinct group. One of the topoisomerase 2 inhibitor clusters grouped next to compounds whose mechanism of action involves double-stranded DNA breaks, suggesting that the ability to produce double-stranded DNA breaks may be a particularly important feature of the mechanism of action in this group. Thus gene expression profiling may provide insights into the mechanism of action of novel drugs, as well as clarify the mechanism of action or metabolism of known drugs.

\section{Nonsense-Mediated RNA Decay Blockade Microarrays}

DNA microarrays can also be harnessed to discover new tumor suppressor genes with mutations in cancer cells. The nonsense-mediated RNA decay (NMD) pathway is an intrinsic cellular mechanism designed to eliminate RNA transcripts containing premature termination codons due to nonsense mutations $(23,24)$. By inhibiting this mechanism, Noensie and Dietz (25) were able to use DNA microarrays to search on a whole-genome level for RNA transcripts whose levels can increase upon NMD inhibition, revealing those genes that contained nonsense mutations. Such a strategy can be applied to the detection of novel truncating mutations in genes in cancer cells, thus enabling the high-throughput discovery of putative tumor suppressor genes. We have used this approach and found it to give a significant enrichment for nonsense mutated transcripts.

\section{Methylation Chips}

Heritable epigenetic alterations may have profound effects on gene transcription and therefore cellular function. Aberrant DNA methylation at promotor regions of critical genes, such as the tumor suppressor gene p16, as well as throughout the genome, has been reported in cancer cells (26). Recently, a high-throughput microarray-based technique was developed to screen thousands of $\mathrm{CpG}$ islands for hypermethylation (27). Novel epigenetic alterations that may affect cell function can reveal novel drug targets whose methylation status may correlate with different tumor epigenotypes.

\section{Comparative Genomic Hybridization Microarrays}

Chromosomal comparative genomic hybridization (CGH), which was first described by Kallioniemi et al. (28), has revolutionized cancer research by allowing one to scan the entire cancer genome for regions of DNA amplification and loss. Although this technology has been widely implemented to reveal many important commonly rearranged loci in cancer genomes, the resolution of chromosomal CGH is only about 10 megabases, a genomic fragment that could code for hundreds of gene candidates that may be affected. DNA microarrays can also be used for comparative hybridization of genomic DNA from two sources, (e.g., tumor and normal) to characterize DNA copy number alterations present in cancer cells but with much higher resolution. The first platform for such an analysis involved the hybridization of genomic DNA to microarrays containing bacterial artificial chromosomes spanning across the genome ("BAC arrays") (29). This approach confirmed previously known loci of DNA copy number changes and identified novel sites of amplification and deletion. This information can be combined with genomic data on gene sites to identify genes that may be driving the deletions and amplifications. On the other hand, a more direct approach is to perform DNA and cDNA hybridization on the same cDNA microarrays, as described by Pollack et al. (30), thus obtaining both DNA copy number information and gene expression data at single gene resolution on the same set of genes on a microarray. In some cases, specialized cDNA microarrays can be constructed with complete coverage of all genes in an amplified loci, allowing for a detailed characterization of the borders of the amplicon and comprehensive analysis of the amplified genes whose overexpression may be causally involved in the clonal selection of cells with that amplification $(31,32)$. Using array $\mathrm{CGH}$, new amplicons and deletions can be identified and a better understanding can be developed of the actual genes targeted and effected by genomic amplification and deletion on a global scale (33-42). For example, a genome-wide study of the effect of gene copy number on gene expression was performed using combined cDNA and CGH microarray analysis of 14 breast cancer cell lines (40) (Figure 1). New overexpressed genes at chromosomal sites previously known to be amplified by standard chromosomal techniques were identified, including 1q21 (6 genes), 8q24 (10 genes), 17q12 (7 genes), 17q 23 (16 genes), and 20q13 (21 genes). New amplicons containing overexpressed genes were also discovered, including three genes overexpressed in a novel amplicon at $17 \mathrm{q} 22$. This amplicon contains the HoxB7 transcription factor, which was then found to be 
overexpressed in a subset of breast cancers (40). These studies highlight the power and increased sensitivity of array CGH combined with cDNA microarrays on the same sample to uncover previously unknown areas of genomic amplification and the genes targeted by these amplifications.

\section{Single Nucleotide Polymorphism Chips}

Besides gene expression profiling, microarray chips can be used to screen for gene polymorphisms on a genome-wide basis. Lindblad-Toh et al. (43) reported an early demonstration of screening tumor DNA for loss of heterozygosity at 15,000 single nucleotide polymorphism (SNP) loci using high-density oligonucleotide arrays. The high-throughput detection of allelic imbalance will reveal new areas of genomic deletions, which are indicators of loci that may contain putative tumor suppressor genes, and thus possible targets for pharmacologic intervention. On the other hand, high-throughput microarray-based SNP assays can also be used to reveal biologically relevant gene polymorphisms that may affect drug metabolism and/or efficacy (44). Since many chemotherapeutic drugs are metabolized to active compounds upon administration to patients, pharmacogenetic variation in drug-metabolizing enzymes due to functionally important polymorphisms in the genes coding for these enzymes may be predictive of response to chemotherapies. Such knowledge will allow the optimal selection of patients for clinical trials that may be genetically more sensitive to the effects of particular agents. With this information, a clinician would then modify or optimize drug selection or dose to ensure maximal therapeutic response.

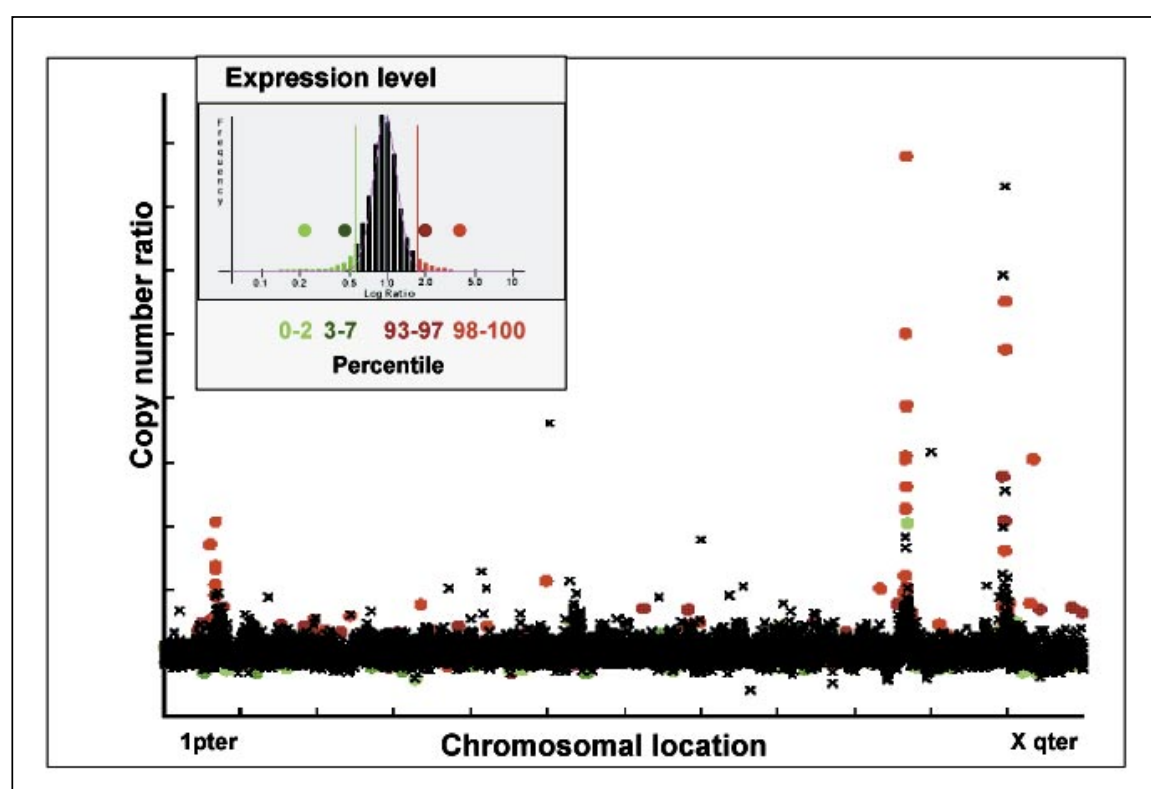

Figure 1. Array CGH/cDNA plot for MCF-7 breast cancer cell line. Array CGH plot for 14,000 genes in MCF-7 breast cancer cell line displayed in chromosomal order from chr 1 pter to chr X qter on the right. Y-axis represents DNA copy number in cell line DNA compared to DNA from normal human white blood cells. Individual data points are labeled according to RNA expression levels (cDNA microarray expression ratios) by color coding according to DNA expression ratios: bright red, upper $2 \%$ of expression ratios (most overexpressed genes); dark red, next $5 \%$ of expression ratios (overexpresssed genes); bright green dots, lowest $2 \%$ of expression ratios (most underexpressed genes); dark green dots, next $5 \%$ of expression ratios (underexpressed genes); and other clones are shown with black crosses.

\section{HIGH-THROUGHPUT CLINICAL TARGET VALIDATION USING TISSUE MICROARRAYS}

The use of DNA microarrays allows the powerful interrogation of thousands of genes for changes in RNA expression, DNA copy number, and the presence of polymorphisms and mutations. The major problem now is how to deal with the avalanche of data generated and, specifically, how to translate this data into clinically effective therapeutics. This is a critical question because of the very high cost and very low efficiency of bringing a drug against a specific target through development and to the clinic. The drug development process is estimated to cost over $\$ 800$ million for each drug and may take over 10 years to be completed. How can one correctly predict from the thousands of candidate targets the few whose pharmaceutical manipulation will impact on cancer?

The first question is that of clinical relevance. Most of the experimental work in drug target discovery is performed on cancer cell lines. To translate these potentially artificial findings to the clinic, and also to assess the impact drugs developed against specific targets are likely to have, it is necessary to know the frequency of the alterations in the candidate gene in clinical samples and the extent of such changes. Moreover, to appreciate the clinical relevance of a candidate target, (he morphological, cellular, and subcellula chene disease-causing alterations in the target ies require examination of large resources of clinical material in collaboration with pathology departments. Even if the use of sophisticated data mining methods allows one to narrow down the list to 20 or even 10 candidate gene targets, their full clinical validation remains a significant hurdle for most genomic laboratories.

A solution to the problem of clinical validation is to assemble pathological samples on a very small scale on a microarray platform that enables parallel analysis [i.e., tissue microarrays (TMAs) (45)]. TMAs are miniaturized collections of hundreds or even thousands of arrayed tissue spots on a glass slide that provide a template for highly parallel validation of specific molecular targets. The use of TMAs allows the high-throughput evaluation of clinically relevant factors associated with the presence of molecular alterations, such as specific features of tissue, cellular and subcellular morphology, and clinical correlates such as patient outcome.

The construction of TMAs is quite simple: cylindrical core biopsies are punched from preselected areas on paraffin-embedded fixed tissue blocks (the kind found in any pathology department). These core biopsies are then inserted in an array format into a recipient paraffin block (Figure 2). Detailed 
technical information on the construction of the TMAs was recently reviewed by Brand et al. (46). Over 1000 individual tissue biopsies can be arrayed onto a recipient block, from which up to 300 thin sections can then be cut with a regular microtome. Each of these sections has the same configuration of tissue spots (rows and columns). TMA slides can be applied for analyses of DNA, RNA, and protein targets, using fluorescence in situ hybridization (FISH), mRNA in situ hybridization, or immunohistochemistry (IHC), respectively. Indeed, one may interrogate with all three methods a virtually identical cohort of tissue samples using a series of serially sectioned TMA slides. Because of the small (0.6- $\mathrm{mm})$ size of the core biopsies, it is feasible to take several biopsies from each donor paraffin block to construct replicate TMA blocks without destroying the original block. For instance, if one creates 10 replicate TMA blocks from 300 tissue samples, the resulting 3000 TMA slides would contain 900,000 total tissue spots available for probing. TMAs therefore enable largescale clinical studies on a single microscope slide. If matching clinical data such as survival and treatment response exists for these specimens, rapid extraction of clinicopathological correlates in these specimens can be performed in a single TMA experiment. Moreover, data from multiple genes can be obtained across that population to determine patterns of involvement among related genes and gene products. Thus, all of the members of a cellular pathway can be studied on successive TMA slides. Another example is the "progression TMA" in which multiple tissue samples at different stages of a disease can be arrayed on one TMA to permit rapid determination of the onset of a molecular event in relationship to the stages of disease progression. It is thus clear that the throughput and uniformity of TMAs can be used for a variety of creative applications to produce data of a scale, quality, and nature that is unique to this platform.

TMAs can be used for the high-throughput analysis of a variety of specimens including multiple tissue and organ types from any disease and normal state. With the current proliferation of lists of candidate genes generated by DNA microarrays, TMAs have already been used to validate and prioritize molecular targets in a variety of ways, including clinical validation in patient tissue samples of results obtained from the analysis of cell lines or rodent disease model systems in vivo, extension of results obtained from the analysis of a limited number of tissue samples by cDNA microarrays to an epidemiologically representative cohort by TMAs, assessment of the prevalence of molecular alterations at various stages of tumor progression, correlation of molecular data with clinicopathological and patient outcome variables, and determination of the cellular and subcellular distribution of the targets.

Three examples of such uses are mentioned here. In a study using cDNA microarrays, Bärlund et al. (47) reported that the ribosomal protein S6 kinase gene is one of several significantly overexpressed and amplified genes in breast cancer cell lines. TMAs containing over 600 clinical breast cancers demonstrated that this gene is amplified and highly expressed at the protein level in $10 \%-15 \%$ of primary breast tumors. Furthermore, concomitant overexpression and amplification of the S6 kinase gene was found to be a significant poor prognostic indicator in this group of breast cancers. In another study, Ginestier et al.
(48) compared mRNA expression levels on cDNA microarrays with protein expression on TMAs for 15 molecules with a proven or suspected role in breast cancer in 55 breast tumors. A good correlation was found only in five of these, thus underlining the necessity for confirming cDNA microarray findings. A TMA of 600 breast tumors was used to identify a prognostic value for one of the molecules, MUC1. This study highlights the need to combine these microarray technologies to obtain clinically useful and reliable information. Finally, Mousses et al. (49) used TMAs to validate differentially expressed genes in hormone refractory versus sensitive prostate cancer xenografts: S100P mRNA expression was increased in xenografts, while CRYM and LMO4 mRNA expression were decreased. A prostate cancer progression microarray was probed with antibodies against each of the three gene products. S100P protein expression was directly correlated with stage of disease, while levels of CRYM and LMO4 proteins were both decreased in a significant number of advanced hormone refractory tumors compared to a population of primary tumors, thereby validating in the clinical context the trends observed in the xenografts.

These studies are but some of the many examples that illustrate the power of the TMA technology for rapid translation of cDNA microarray results into clinically meaningful information. An analysis of hundreds of tumor samples can

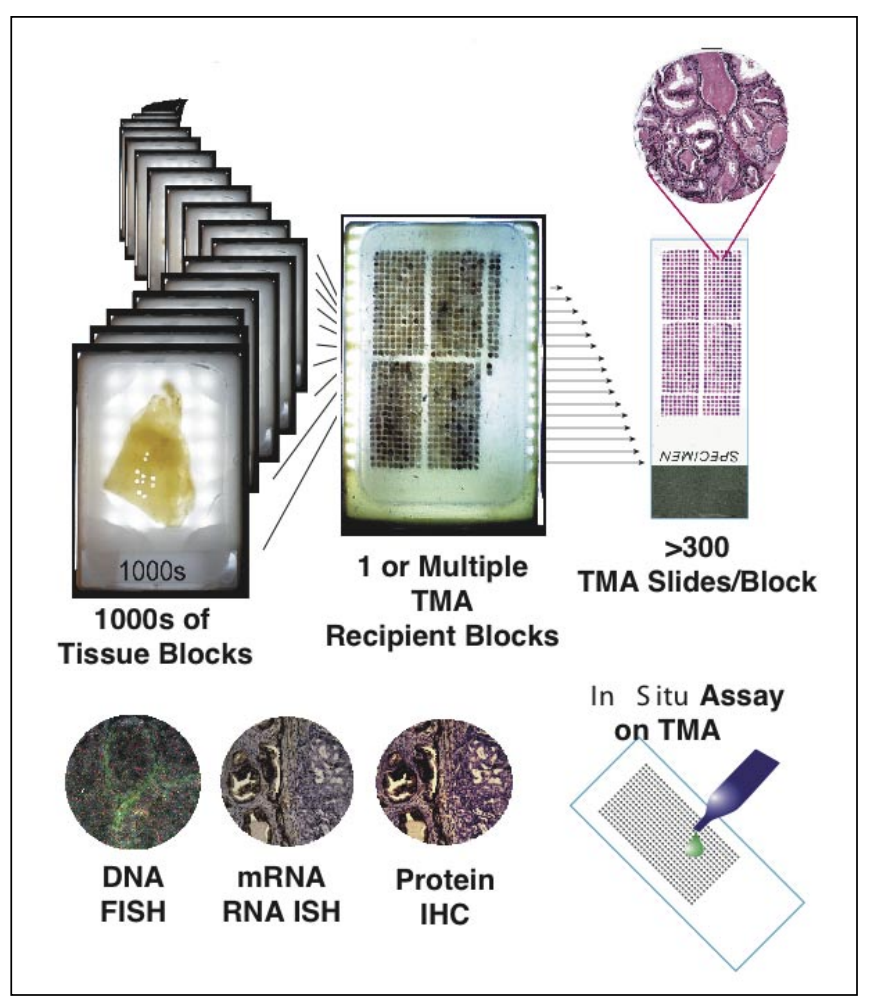

Figure 2. Tissue microarray technology. Thousands of paraffin-embedded fixed tissue blocks are selected for core biopsies, which are placed into an array format onto a recipient paraffin block. The recipient block is then sectioned over 300 times and the sections placed onto microscope slides. Each slide has the same tissues in the same coordinates as the recipient block. These slides can then be used for in situ assays including fluorescence in situ hybridization (FISH), RNA in situ hybridization (RNA ISH), and protein immunohistochemistry (IHC) (modified from Hum. Mol. Genet. 10:657-662, 2001). TMA, tissue microarrays. 
be performed within a few weeks - a task that would otherwise have taken years to accomplish using traditional pathological techniques. We predict that this powerful research approach will be increasingly applied in the future, as more and more investigators seek the validation and prioritization of their early cDNA microarray leads.

\section{HIGH-THROUGHPUT CHARACTERIZATION OF GENE FUNCTION USING CELL BIOCHIPS}

Alterations in gene or protein expression levels do not provide information about the biological importance of the gene or its suitability as a drug target. Simultaneously with clinical validation, it is necessary to "functionally" validate target genes obtained from microarray experiments (i.e., to verify whether the observed molecular changes are responsible for significant phenotypic or functional changes in the tissue of interest). Functional validation is traditionally performed on a gene-by-gene basis. Assays often have to be specifically designed for each candidate target. For example, investigators may screen for the phenotypic effects of gene overexpression by knocking down gene expression. Specific biochemical assays such as assays for enzymatic activity may have to be developed for some targets to search for small molecule inhibitors (50) from compound libraries. Such high-throughput

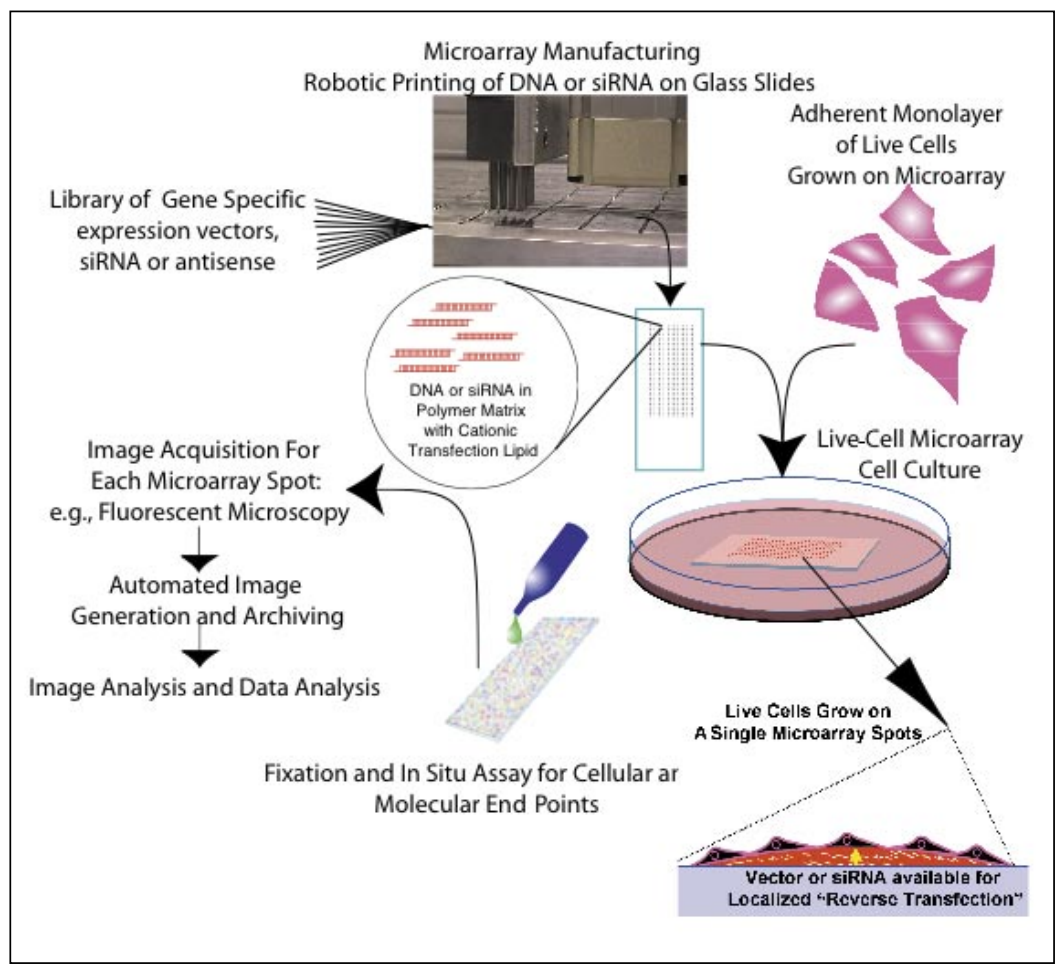

Figure 3. Live-cell microarray technology. Live-cell arrays are created starting with a library of vectors or small interfering RNAs (siRNAs) that are printed onto glass slides within a polymer matrix containing cationic lipid transfection agent. Slides are placed into a tissue culture dish onto which live cells are placed to grow as a monolayer on the slides. The cells growing over the spotted vectors or siRNAs are transfected and are assayed for cellular and molecular end points of interest. Finally, image acquisition by fluorescent microscopy is followed by image analysis and archiving. screening has usually been carried out in a microtiter plate format for each gene target, but the abundance of targets arising from genomics and proteomics surveys will require parallel approaches to rapidly investigate their function.

Recently, a well-less microarray platform was proposed for high-throughput functional characterization. "Reverse transfection," as described by Ziauddin and Sabatini (51), enables parallel transfection of hundreds of genes in a microarray format. Plasmid expression vectors containing full-length cDNAs were complexed with a lipid transfection reagent and printed at a high density on a microscope glass slide, over which adherent cells are grown. Cells growing over the DNA spots are transfected by the vectors, while the other cells are not, resulting in expression of specific proteins in spatially distinct groups of cells (Figure 3). Various phenotypic effects of this reverse transfection of hundreds of genes can be detected using specific cell-based bioassays.

This cell-based array system using cDNAs as transgenes can be used to identify drug-target interactions. The power of this technology lies in the parallel nature and miniaturization of gene transfer into live cells for high-throughput analysis of the molecular and the phenotypic effects of specific transgenes.

An alternative approach employing live-cell, microarraybased technology is high-throughput specific silencing of genes. Sequence-specific inhibition of gene expression can be accomplished either using single-stranded antisense oligonucleotides or small interfering RNAs (siRNAs). siRNA are RNA duplexes (52-55) that trigger a recently identified mechanism termed RNA interference (RNAi), which leads to potent gene silencing. Many researchers are now routinely using siRNAs to knock down specific genes to study their function. We have performed proofof-principle, live-cell, RNAi-based microarray experiments that demonstrate sequence specific and spatially confined siRNA-induced gene silencing on a well-less platform. RNAi microarrays are ideal for functional screening and parallel biological analysis and may have an advantage over arrays making use of transgene expression: overexpression of a given gene may not generate a physiologically relevant phenotype, whereas the inhibition of gene expression has proven to be a highly successfully method for delineating gene function.

Although efforts are under way to generate human genome-wide libraries of siRNAs (55), these reagents are likely to be costly and their plate-based analysis expensive and timeconsuming. RNAi-based microarrays have the advantage of requiring significantly less material than conventional well-based systems and can be easily adapted for a broad range of functional, high-throughput, cell-based assays. While the live-cell microarray technologies require further development, their potential for enabling genomic-scale functional analysis could significantly speed up our ability to link associative gene expression data with a func- 
tional effect. One of the biggest challenges for either type of live-cell array will be extracting quantitative data from the cells on the microarray spots. Fortunately, the development of imaging systems for tissue microarray analysis can be directly applied to imaging of cell microarrays treated with various stains and assays. For example, a fluorescent microscopy system fitted with automated stage control for high-throughput fluorescent image acquisition of DNA FISH of tissue microarrays can easily be adapted and utilized for capturing images from fluorescent end points on cells sitting on cell transfection microarrays. Similarly, data management systems developed for tissue microarray images and image analysis can be directly modified and adapted for the needs of cell transfection microarrays.

\section{CONCLUSIONS}

High-throughput genomic and proteomic screening technologies have led to a massive increase in the rate of data generation in the cancer research field, greatly exceeding the rate at which biological significance and clinical relevance can be determined. The consequence of the new discovery technologies is that the validation of targets has become the rate-limiting step in translating genomic and proteomic information to clinical and cancer therapeutic applications. This limitation has consequently hindered the new biological insight and medical discoveries that were expected to result from the completion of the Human Genome Project. In this review, we presented a sampling of new and emerging highthroughput technologies to discover, validate, and translate genomic information. Although the examples of solid phase platforms presented differ in many ways, the DNA, tissue, and live-cell transfection microarrays have some common unifying themes, including high throughput, miniaturization, and parallel data generation. Each of these technologies is powerful and useful, but to truly accelerate anticancer drug discovery, these technologies need to be incorporated into a systems biology process that permits all of the discoveries to be advanced in parallel. Such integration of different microarray-based approaches will enable translational genomics systems that are capable of greatly increasing the flow of information from the genome to the bedside.

\section{ACKNOWLEDGMENTS}

We thank Olli Kallioniemi, Anne Kallioniemi, Natasha Caplen, and David Azorsa who have contributed directly and indirectly through numerous conversations about the concepts and ideas related to the application and integration of high-throughput technologies.

\section{REFERENCES}

1.Schena, M., D. Shalon, R.W. Davis, and P.O. Brown. 1995. Quantitative monitoring of gene expression patterns with a complementary DNA microarray. Science 270:467-470.

2.Lockhart, D.J., H. Dong, M.C. Byrne, M.T. Follettie, M.V. Gallo, M.S.
Chee, M. Mittmann, C. Wang, et al. 1996. Expression monitoring by hybridization to high-density oligonucleotide arrays. Nat. Biotechnol. 14: $1675-1680$

3.Schena, M., D. Shalon, R. Heller, A. Chai, P.O. Brown, and R.W. Davis. 1996. Parallel human genome analysis: microarray-based expression monitoring of 1000 genes. Proc. Natl. Acad. Sci. USA 93:10614-10619.

4.DeRisi, J.L., V.R. Iyer, and P.O. Brown. 1997. Exploring the metabolic and genetic control of gene expression on a genomic scale. Science 278: 680-686.

5.DeRisi, J., L. Penland, P.O. Brown, M.L. Bittner, P.S. Meltzer, M. Ray, Y. Chen, Y.A. Su, and J.M. Trent. 1996. Use of a cDNA microarray to analyse gene expression patterns in human cancer. Nat. Genet. 14: 457-460.

6.Golub, T.R., D.K. Slonim, P. Tamayo, C. Huard, M. Gaasenbeek, J.P. Mesirov, H. Coller, M.L. Loh, et al. 1999. Molecular classification of cancer: class discovery and class prediction by gene expression monitoring. Science 286:531-537.

7.Perou, C.M., T. Sorlie, M.B. Eisen, M. van de Rijn, S.S. Jeffrey, C.A. Rees, J.R. Pollack, D.T. Ross, et al. 2000. Molecular portraits of human breast tumours. Nature 406:747-752.

8.Bittner, M., P. Meltzer, Y. Chen, Y. Jiang, E. Seftor, M. Hendrix, M. Radmacher, R. Simon, et al. 2000. Molecular classification of cutaneous malignant melanoma by gene expression profiling. Nature 406:536-540.

9.Weeraratna, A.T., Y. Jiang, G. Hostetter, K. Rosenblatt, P. Duray, M. Bittner, and J.M. Trent. 2002. Wnt5a signaling directly affects cell motility and invasion of metastatic melanoma. Cancer Cell 1:279-288.

10.Hedenfalk, I., D. Duggan, Y. Chen, M. Radmacher, M. Bittner, R. Simon, P. Meltzer, B. Gusterson, et al. 2001. Gene-expression profiles in hereditary breast cancer. N. Engl. J. Med. 344:539-548.

11.Nielsen, T.O., R.B. West, S.C. Linn, O. Alter, M.A. Knowling, J.X. O'Connell, S. Zhu, M. Fero, et al. 2002. Molecular characterisation of soft tissue tumours: a gene expression study. Lancet 359:1301-1307.

12.Virtanen, C., Y. Ishikawa, D. Honjoh, M. Kimura, M. Shimane, T. Miyoshi, H. Nomura, and M.H. Jones. 2002. Integrated classification of lung tumors and cell lines by expression profiling. Proc. Natl. Acad. Sci. USA 99:12357-12362.

13.Dyrskjot, L., T. Thykjaer, M. Kruhoffer, J.L. Jensen, N. Marcussen, S. Hamilton-Dutoit, H. Wolf, and T.F. Orntoft. 2003. Identifying distinct classes of bladder carcinoma using microarrays. Nat. Genet. 33:90-96.

14.Schwartz, D.R., S.L. Kardia, K.A. Shedden, R. Kuick, G. Michailidis, J.M. Taylor, D.E. Misek, R. Wu, et al. 2002. Gene expression in ovarian cancer reflects both morphology and biological behavior, distinguishing clear cell from other poor-prognosis ovarian carcinomas. Cancer Res. 62: 4722-4729.

15.Fuller, G.N., K.R. Hess, C.H. Rhee, W.K. Yung, R.A. Sawaya, J.M. Bruner, and W. Zhang. 2002. Molecular classification of human diffuse gliomas by multidimensional scaling analysis of gene expression profiles parallels morphology-based classification, correlates with survival, and reveals clinically-relevant novel glioma subsets. Brain Pathol. 12:108116.

16.Okutsu, J., T. Tsunoda, Y. Kaneta, T. Katagiri, O. Kitahara, H. Zembutsu, R. Yanagawa, S. Miyawaki, et al. 2002. Prediction of chemosensitivity for patients with acute myeloid leukemia, according to expression levels of 28 genes selected by genome-wide complementary DNA microarray analysis. Mol. Cancer Ther. 1:1035-1042.

17.Scherf, U., D.T. Ross, M. Waltham, L.H. Smith, J.K. Lee, L. Tanabe, K.W. Kohn, W.C. Reinhold, et al. 2000. A gene expression database for the molecular pharmacology of cancer. Nat. Genet. 24:236-244.

18.Staunton, J.E., D.K. Slonim, H.A. Coller, P. Tamayo, M.J. Angelo, J. Park, U. Scherf, J.K. Lee, et al. 2001. Chemosensitivity prediction by transcriptional profiling. Proc. Natl. Acad. Sci. USA 98:10787-10792.

19.Marton, M.J., J.L. DeRisi, H.A. Bennett, V.R. Iyer, M.R. Meyer, C.J. Roberts, R. Stoughton, J. Burchard, et al. 1998. Drug target validation and identification of secondary drug target effects using DNA microarrays. Nat. Med. 4:1293-1301.

20.Hughes, T.R., M.J. Marton, A.R. Jones, C.J. Roberts, R. Stoughton, C.D. Armour, H.A. Bennett, E. Coffey, et al. 2000. Functional discovery via a compendium of expression profiles. Cell 102:109-126.

21.Mousses, S., U. Wagner, Y. Chen, J.W. Kim, L. Bubendorf, M. Bittner, T. Pretlow, A.G. Elkahloun, et al. 2001. Failure of hormone therapy in prostate cancer involves systematic restoration of androgen responsive genes and activation of rapamycin sensitive signaling. Oncogene 20 
6718-6723.

22.Kim, S., E.R. Dougherty, M.L. Bittner, Y. Chen, K. Sivakumar, P. Meltzer, and J.M. Trent. 2000. General nonlinear framework for the analysis of gene interaction via multivariate expression arrays. J. Biomed. Opt. 5:411-424.

23.Losson, R. and F. Lacroute. 1979. Interference of nonsense mutations with eukaryotic messenger RNA stability. Proc. Natl. Acad. Sci. USA 76: 5134-5137.

24.Frischmeyer, P.A. and H.C. Dietz. 1999. Nonsense-mediated mRNA decay in health and disease. Hum. Mol. Genet. 8:1893-1900.

25.Noensie, E.N. and H.C. Dietz. 2001. A strategy for disease gene identification through nonsense-mediated mRNA decay inhibition. Nat. Biotechnol. 19:434-439.

26.Baylin, S.B., J.G. Herman, J.R. Graff, P.M. Vertino, and J.P. Issa. 1998. Alterations in DNA methylation: a fundamental aspect of neoplasia. Adv. Cancer Res. 72:141-196.

27.Yan, P.S., M.R. Perry, D.E. Laux, A.L. Asare, C.W. Caldwell, and T.H. Huang. 2000. CpG island arrays: an application toward deciphering epigenetic signatures of breast cancer. Clin. Cancer Res. 6:1432-1438.

28.Kallioniemi, A., O.P. Kallioniemi, D. Sudar, D. Rutovitz, J.W. Gray, F. Waldman, and D. Pinkel. 1992. Comparative genomic hybridization for molecular cytogenetic analysis of solid tumors. Science 258:818-821.

29.Pinkel, D., R. Segraves, D. Sudar, S. Clark, I. Poole, D. Kowbel, C. Collins, W.L. Kuo, et al. 1998. High resolution analysis of DNA copy number variation using comparative genomic hybridization to microarrays. Nat. Genet. 20:207-211.

30.Pollack, J.R., C.M. Perou, A.A. Alizadeh, M.B. Eisen, A. Pergamenschikov, C.F. Williams, S.S. Jeffrey, D. Botstein, and P.O. Brown. 1999. Genome-wide analysis of DNA copy-number changes using cDNA microarrays. Nat. Genet. 23:41-46.

31.Monni, O., E. Hyman, S. Mousses, M. Barlund, A. Kallioniemi, and O.P. Kallioniemi. 2001. From chromosomal alterations to target genes for therapy: integrating cytogenetic and functional genomic views of the breast cancer genome. Semin. Cancer Biol. 11:395-401.

32.Monni, O., M. Barlund, S. Mousses, J. Kononen, G. Sauter, M. Heiskanen, P. Paavola, K. Avela, et al. 2001. Comprehensive copy number and gene expression profiling of the 17q23 amplicon in human breast cancer. Proc. Natl. Acad. Sci. USA 98:5711-5716.

33.Hodgson, G., J.H. Hager, S. Volik, S. Hariono, M. Wernick, D. Moore, N. Nowak, D.G. Albertson, et al. 2001. Genome scanning with array $\mathrm{CGH}$ delineates regional alterations in mouse islet carcinomas. Nat. Genet. 29:459-464.

34.Wilhelm, M., J.A. Veltman, A.B. Olshen, A.N. Jain, D.H. Moore, J.C. Presti, Jr., G. Kovacs, and F.M. Waldman. 2002. Array-based comparative genomic hybridization for the differential diagnosis of renal cell cancer. Cancer Res. 62:957-960.

35.Hui, A.B., K.W. Lo, P.M. Teo, K.F. To, and D.P. Huang. 2002. Genome wide detection of oncogene amplifications in nasopharyngeal carcinoma by array based comparative genomic hybridization. Int. J. Oncol. 20: 467-473.

36.Cai, W.W., J.H. Mao, C.W. Chow, S. Damani, A. Balmain, and A. Bradley. 2002. Genome-wide detection of chromosomal imbalances in tumors using BAC microarrays. Nat. Biotechnol. 20:393-396.

37.Clark, J., S. Edwards, M. John, P. Flohr, T. Gordon, K. Maillard, I. Giddings, C. Brown, et al. 2002. Identification of amplified and expressed genes in breast cancer by comparative hybridization onto microarrays of randomly selected cDNA clones. Genes Chromosomes Cancer 34:104-114

38.Ishizuka, T., C. Tanabe, H. Sakamoto, K. Aoyagi, M. Maekawa, N. Matsukura, A. Tokunaga, T. Tajiri, et al. 2002. Gene amplification profiling of esophageal squamous cell carcinomas by DNA array CGH. Biochem. Biophys. Res. Commun. 296:152-155.

39.Pollack, J.R., T. Sorlie, C.M. Perou, C.A. Rees, S.S. Jeffrey, P.E. Lonning, R. Tibshirani, D. Botstein, et al. 2002. Microarray analysis reveals a major direct role of DNA copy number alteration in the transcriptional program of human breast tumors. Proc. Natl. Acad. Sci. USA 99:1296312968.

40.Hyman, E., P. Kauraniemi, S. Hautaniemi, M. Wolf, S. Mousses, E. Rozenblum, M. Ringner, G. Sauter, et al. 2002. Impact of DNA amplification on gene expression patterns in breast cancer. Cancer Res. 62 : 6240-6245

41.Clark, J., S. Edwards, A. Feber, P. Flohr, M. John, I. Giddings, S.
Crossland, M.R. Stratton, et al. 2003. Genome-wide screening for complete genetic loss in prostate cancer by comparative hybridization onto cDNA microarrays. Oncogene 22:1247-1252.

42.Albertson, D.G. 2003. Profiling breast cancer by array CGH. Breast Cancer Res. Treat. 78:289-298.

43.Lindblad-Toh, K., D.M. Tanenbaum, M.J. Daly, E. Winchester, W.O. Lui, A. Villapakkam, S.E. Stanton, C. Larsson, et al. 2000. Loss-ofheterozygosity analysis of small-cell lung carcinomas using single-nucleotide polymorphism arrays. Nat. Biotechnol. 18:1001-1005.

44.Liljedahl, U., J. Karlsson, H. Melhus, L. Kurland, M. Lindersson, T. Kahan, F. Nystrom, L. Lind, and A.C. Syvanen. 2003. A microarray minisequencing system for pharmacogenetic profiling of antihypertensive drug response. Pharmacogenetics 13:7-17.

45.Kononen, J., L. Bubendorf, A. Kallioniemi, M. Barlund, P. Schraml, S. Leighton, J. Torhorst, M.J. Mihatsch, et al. 1998. Tissue microarrays for high-throughput molecular profiling of tumor specimens. Nat. Med. 4:844-847.

46.Brand, K., G. Hostetter, O.P. Kallioniemi, J. Kononen, G. Sauter, and M. Trivett. 2003. Tissue microarrays, pp. 608-616. In D. Bowtell and J. Sambrook (Eds.), DNA Microarrays, A Molecular Cloning Manual. Cold Spring Harbor Laboratory Press, Cold Spring Harbor.

47.Barlund, M., F. Forozan, J. Kononen, L. Bubendorf, Y. Chen, M.L. Bittner, J. Torhorst, P. Haas, et al. 2000. Detecting activation of ribosomal protein S6 kinase by complementary DNA and tissue microarray analysis. J. Natl. Cancer Inst. 92:1252-1259.

48.Ginestier, C., E. Charafe-Jauffret, F. Bertucci, F. Eisinger, J. Geneix, D. Bechlian, N. Conte, J. Adelaide, et al. 2002. Distinct and complementary information provided by use of tissue and DNA microarrays in the study of breast tumor markers. Am. J. Pathol. 161:1223-1233.

49.Mousses, S., L. Bubendorf, U. Wagner, G. Hostetter, J. Kononen, R. Cornelison, N. Goldberger, A.G. Elkahloun, et al. 2002. Clinical validation of candidate genes associated with prostate cancer progression in the CWR22 model system using tissue microarrays. Cancer Res. 62: 1256-1260.

50.Vidal, M. and H. Endoh. 1999. Prospects for drug screening using the reverse two-hybrid system. Trends Biotechnol. 17:374-381.

51.Ziauddin, J. and D.M. Sabatini. 2001. Microarrays of cells expressing defined cDNAs. Nature 411:107-110.

52.Caplen, N.J., S. Parrish, F. Imani, A. Fire, and R.A. Morgan. 2001 Specific inhibition of gene expression by small double-stranded RNAs in invertebrate and vertebrate systems. Proc. Natl. Acad. Sci. USA 98 : 9742-9747.

53.Elbashir, S.M., W. Lendeckel, and T. Tuschl. 2001. RNA interference is mediated by 21- and 22-nucleotide RNAs. Genes Dev. 15:188-200.

54.Elbashir, S.M., J. Harborth, W. Lendeckel, A. Yalcin, K. Weber, and T. Tuschl. 2001. Duplexes of 21-nucleotide RNAs mediate RNA interference in cultured mammalian cells. Nature 411:494-498.

55.Hannon, G.J. 2002. RNA interference. Nature 418:244-251.

\section{Address correspondence to:}

Jeffrey Trent

Translational Genomics Research Institute

400 N. 5th Street, Suite 1600

Phoenix, AZ 85004, USA

e-mail: jtrent@tgen.org 